\title{
Cytotoxicity for Porcine Islet Cells by Complement of Six Animal Species
}

\author{
Shin ITAKURA ${ }^{1)}$, Hisamune KATO $^{2)}$, Pi-Chao WANG ${ }^{2)}$, Atsuko MATSUNAGA ${ }^{3)}$, Tomofumi JITSUKAWA ${ }^{3)}$, \\ Kazuya EDAMURA ${ }^{1)}$, Hisako OHGAWARA ${ }^{4)}$, Manabu MOCHIZUKI ${ }^{1)}$, Ryohei NISHIMURA ${ }^{1)}$ and Nobuo SASAKI ${ }^{1)}$ \\ ${ }^{1)}$ Laboratory of Veterinary Surgery, Graduate School of Agricultural and Life Sciences, University of Tokyo, 1-1-1 Yayoi, Bunkyo-ku, \\ Tokyo 113-8657, ${ }^{2}$ Institute of Applied Biochemistry, Tsukuba University, 1-1-1 Tennodai 305-8572, ${ }^{3)}$ Axia Biosciences, Inc., \\ Shinanomachi Campus-Research Park, Keio University School of Medicine, 35 Shinanomachi, Shinjuku-ku, Tokyo 160-8582, and \\ ${ }^{4)}$ Medical Research Institute, Tokyo Women's Medical University School of Medicine, 8-1 Kawada-cho, Shinjuku-ku, Tokyo 162-8666, \\ Japan
}

(Received 6 January 2003/Accepted 16 June 2003)

ABSTRACT. Complement-mediated cytotoxicity for porcine islet cells (PICs) was evaluated using sera of six animal species. Then soluble complement receptor type-1 (sCR1) as an anti-complement agent was added to those sera, and the changes in 50\% hemolytic unit of complement serum (CH50) and cytotoxic effect of those sera on PICs were examined. All the sera except for that of pig showed cytotoxicity. However, the extent of toxicity was considerably different between species. In the rat and human serum, sCR1 significantly reduced $\mathrm{CH} 50$ and cytotoxicity, however in the dog serum, sCR1 had no suppressive effects. These results may suggest that complement contribute to humoral cytotoxicity for PICs as a main factor, and the compatibility of complement with PICs differs between anim al species.

KEY WORDS: complement, cytotoxicity, porcine islet cell.

J. Vet. Med. Sci. 65(10): 1107-1109, 2003

Xenotransplantation of porcine islet cells (PICs) has been considered to be one of the alternatives to whole pancreas transplantation for the treatment of patients with type- 1 diabetes mellitus. For the successful xenotransplantation, the immune response to the xenotransplanted islet cells by the recipients must be controlled. Hyperacute rejection (HAR) of xenografts mediated by the activation of complement system is a well-known process in organ transplantation, which results in the loss of the graft within a few min to hr of transplantation $[2,10]$. However the role of HAR in rejection of xenotransplanted PICs is still not completely understood. Recent researches on HAR in the xenotransplantation of the pig-to-human model in vitro suggest that humoral cytotoxicity for PICs is mediated by activation of complement triggered mainly by natural antibody bonding to the cells [12]. On the other hand, it has been reported that sugar antigen Galactose Alpha 1-3 Galactose (Gal $\alpha(1-3) \mathrm{Gal})$, which is considered to be a major factor in initiating HAR for vascularized organ xenografts, may not be expressed on the surface of adult PICs [4, 9]. Thus PICs are considered to be destroyed by polyclonal anti-pig antibodies and complements [6].

Although complement is related to humoral cytotoxicity in pig-to-human islet transplantation models in vitro, there have been no researches investigating a characteristic of complement-mediated cytotoxicity for PICs by using sources of complement from other animal species. The purpose of this study is to examine the humoral cytotoxicity for isolated PICs using sera of several animal species, and to investigate the participation of complements in the rejection of PICs by humoral factors.

Adult pig pancreas was used as a source of PICs. The method of isolation of PICs was described previously $[1,8$,
11]. Briefly, adult pig pancreases were obtained from the local slaughterhouse. The pancreas was immediately trimmed and minced with scissors after slaughtered as soon as possible, then was digested by gentle stirring in phosphate buffered saline (PBS) supplemented with 3\% newborn calf serum and incubated at $27^{\circ} \mathrm{C}$ without exogenous enzymes. Islet cells in PBS were collected by centrifugation and purified with Histopaque-1077 (Sigma, St. Louis, MO, U.S.A.). The purified islet cells were washed three times and resuspended in RPMI 1640 medium supplemented with $10 \mathrm{mM}$ nicotinamide (Wako, Osaka, Japan) and $10 \%$ fetal bovine serum.

Sera of the rabbit, guinea pig, rat, dog, pig and human were used as sources of complement. Sera of rabbit, guinea pig and rat were purchased as Rabbit Complement, Guinea Pig Complement (ICN Pharmaceuticals Inc., Aurora, OH, U.S.A.) and Rat Complement (Inter-Cell Technologies Inc., Somerville, NJ, U.S.A.), respectively. Dog sera were obtained from 5 healthy Beagle dogs, human sera from 5 healthy donors, and pig sera from 5 slaughtered pigs. All the sera were pooled and stored in aliquots at $-80^{\circ} \mathrm{C}$. Complement activity of all sera was assessed by measuring $50 \%$ hemolytic unit of complement serum (CH50) by sheep red blood cell hemolytic titration [5] immediately before the following experiments.

Serum cytotoxicity for PICs was evaluated using Cell Counting Kit (Dojindo, Osaka, Japan) where 2-(2-methoxy4-nitrophenyl)-3-(4-nitrophenyl)-5-(2,4-disulfophenyl)-2Htetrazolium, monosodium salt (WST-8) was used as a reagent. The cultured PICs were resuspended in the culture medium and seeded in triplicate in a 96-well plate at a density of $5 \times 10^{4}$ cells/well. The medium was discarded after PICs were incubated for $48 \mathrm{hr}$ and adhered to a bottom of 
the plate, then the serum of each animal diluted to a final concentration of $12.5 \%$ with $100 \mu \mathrm{l}$ culture medium was added to each well. Heat-inactivated serum diluted to the same concentration as the corresponding intact serum with $100 \mu l$ culture medium was also used as a negative control. PICs were incubated with serum at $37^{\circ} \mathrm{C}$ for $24 \mathrm{hr}$, then WST-8 was added to each well. After a 4-hr incubation, optical density (OD) at $490 \mathrm{~nm}$ was measured by a microplate spectrometer (Bio-Rad Laboratories Inc., Tokyo, Japan) with $630 \mathrm{~nm}$ as a reference wavelength. The percentage of survival of PICs was calculated by the following equation: $\%$ islet cells survival $=\{100-(a-p) /(a-m)\} \times 100$ ( $a=O D$ of control well, $\mathrm{p}=\mathrm{OD}$ of test well, $\mathrm{m}=\mathrm{OD}$ of background).

In order to investigate the participation of complement in serum cytotoxicity for PICs, a suppressive effect of soluble complement receptor type-1 (sCR1) on CH50 and cytotoxicity for PICs of the sera was examined. Because the available volume of sCR1 was limited, only the sera of human, dog and rat were used in this experiment, since these animal species would be used in our further study for PICs transplantation. sCR1 was produced by the cultivation of Chinese Hamster Ovary cells transfected with human sCR1 gene. The secreted SCR1 was purified by two-stage purification by chromatography. The detail was reported elsewhere [3]. Serial concentrations of $\operatorname{sCR} 1(0,50,100$, and $300 \mu \mathrm{g}$ ) were prepared and added to $1 \mathrm{~m} l$ of the serum of each animal and the change in $\mathrm{CH} 50$ was measured. In the next experiment, the dog, rat, and human sera were added with different doses of sCR $1(50,100$, and $300 \mu \mathrm{g} / \mathrm{m} l$ of serum). Then the dog and rat sera were diluted to the concentration of $12.5 \%$, and the human sera to $50 \%$ with the medium. Heat-inactivated sera diluted to the same concentration as that of each animal were used as negative control. PICs were seeded on a 96-well plate as above in the cytotoxicity assay, then $100 \mu \mathrm{l}$ of the diluted serum was added to each well. The percentage of survival was obtained using Cell Counting Kit (Dojindo) in the same manner as described above. For comparison of the change in survival rates with each concentration of sCR1 and without sCR1, one-factor analysis of variance followed by Fisher's PLSD test was used, and p values less than 0.05 were considered significant.

Sera of all animal species except for the pig serum showed cytotoxicity on PICs. However the degree of toxicity was considerably different between species (Fig. 1). Dog and rabbit sera exerted strong cytotoxicity at the lower serum concentration (12.5\%) against PICs. In the dog serum, survival rate of PICs against control was $36.9 \pm$ $8.9 \%$, and in the rabbit serum, it was $57.9 \pm 6.4 \%$. On the contrary, the human serum showed minimal cytotoxicity at the lower serum concentrations $(12.5 \%$ and $25 \%)$, and even in the high concentration $(50 \%)$ a survival rate was $90.7 \pm$ $1.5 \%$. The guinea pig serum showed cytotoxicity with survival rates of $87.5 \pm 60.9 \%, 86.1 \pm 63.2 \%$ at serum concentrations of 25 and $50 \%$, respectively. The rat serum showed cytotoxicity with a survival rate of $79.9 \pm 67.5 \%$ at a serum

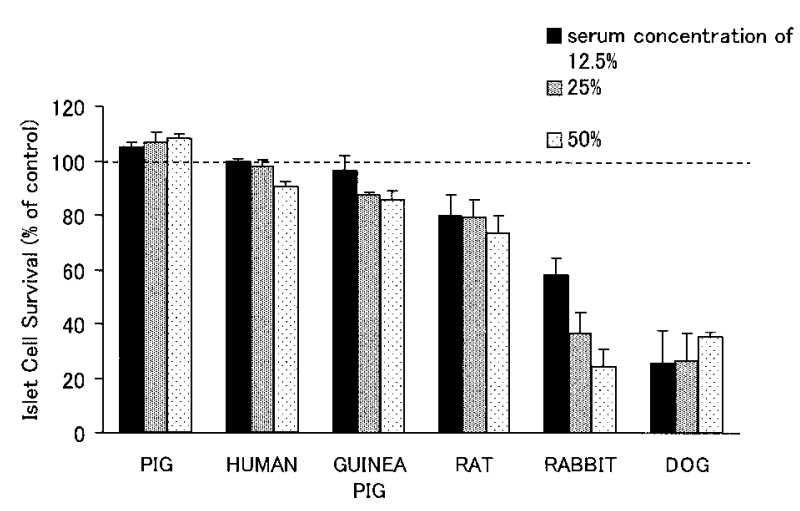

Fig. 1. Cytotoxicity for porcine islet cells (PICs) by the sera of six animal species. Isolated PICs were incubated with three concentrations of each serum. Heat-inactivated serum of each species with each concentration was used as a negative control. Results are expressed as mean values of triplicates \pm standard deviation (SD) when the viable cell number of the control was set at $100 \%$.

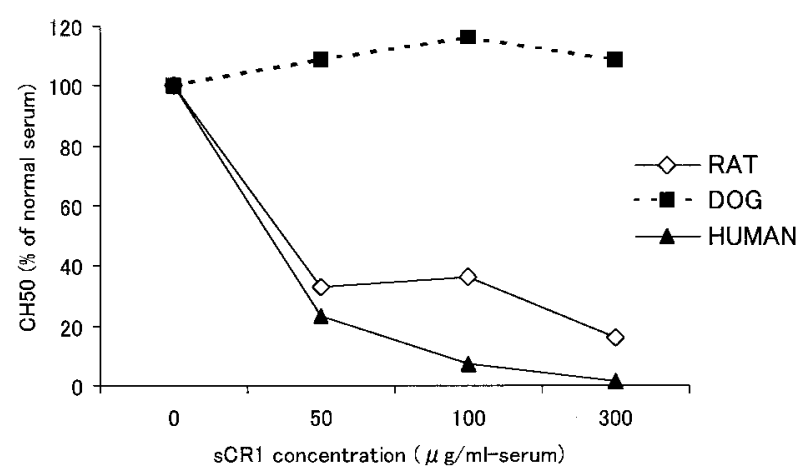

Fig. 2. Percent changes in 50\% hemolytic unit of complement serum (CH50) of the dog, rat, and human sera after soluble complement receptor type-1 (sCR1) treatments of different concentrations comparing to that of the control serum without sCR1 $(100 \%)$.

concentration of $12.5 \%$. Pig serum did not show any cytotoxic effects on allogenic PICs.

The changes in $\mathrm{CH} 50$ of the dog, rat, and human sera when sCR1 was treated were shown in Fig. 2. Data were expressed as a percentage against those without sCR1. A suppressive effect of sCR 1 on $\mathrm{CH} 50$ was demonstrated when it was added to the human and rat sera. $\mathrm{CH} 50$ was suppressed to $15.8 \%$ in the rat serum and to $1.2 \%$ in the human serum of the non-added level at the sCR1 concentration of $300 \mu \mathrm{g} / \mathrm{m} l$ serum. However in the dog serum, no suppressive effect on $\mathrm{CH} 50$ was observed. Similar results were obtained in the cytotoxicity assay with SCR1, as shown in Fig. 3, in which the percentage islet survival of the rat and human groups was increased in a dose dependent manner when SCR 1 was added. A significant suppressive effect was detected in the rat serum when sCR1 was added as compared to that without sCR1. In the dog serum, no suppressive effect was recognized in this cytotoxicity assay. 


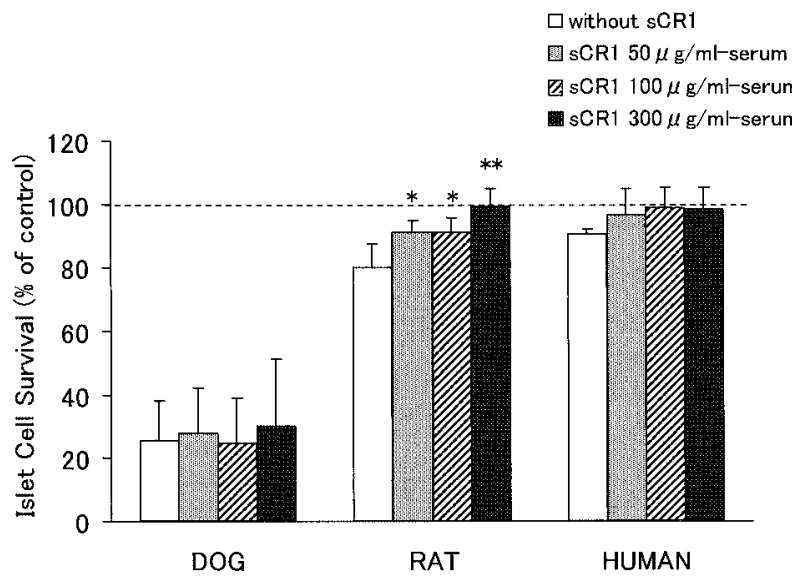

Fig. 3. Changes in cytotoxicity for PICs by adding sCR1 of different concentrations to the three sera. Heat-inactivated serum of each species was used as a negative control. Results are expressed as mean values of triplicate $\pm \mathrm{SD}$ when the viable cell number of the control was set at $100 \%$. ${ }^{*} \mathrm{p}<0.05, * * \mathrm{p}<0.01$ for statistical difference between the serum without sCR1 and the serum treated with sCR1.

In the present study, we focused on the compatibility of PICs to humoral immune factors of several animal species. There was a great difference in serum cytotoxicity for PICs among animal species. The result revealed that the dog serum was the most incompatible with PICs among others, whereas the human serum was the most compatible. In addition, the cytotoxicity was suppressed by the addition of sCR1 to the sera of each animal except for the dog serum. The reason for the ineffectiveness of $\mathrm{sCR} 1$ on the prevention of cytotoxicity of the dog serum is unclear. sCR1 used in this study was originated from human one, therefore the structural incompatibility between human sCR1 and dog complement may be a factor of the ineffectiveness. Furthermore, there was a correlation between suppressive effect of sCR 1 on $\mathrm{CH} 50$ and cytotoxicity. These results may suggest that the serum cytotoxicity for PICs was mainly mediated by complement, although the hypothesis in this study was that complement-mediated cytotoxicity would not act on adult PICs because of the lack of Gal $\alpha(1-3) \mathrm{Gal}$ on them. This may be suggest that other mechanisms to activate the complement system such as other antigenic sugar antigen other than Gal $\alpha(1-3) \mathrm{Gal}$ may be involved in the cytotoxicity to PICs. Or other unknown components, which had toxic effect on PICs, could not be completely eliminated in this study.

Compatibility of complement with complement regulatory proteins expressed on the cell surface, such as decay accelerating factor (DAF), membrane cofactor protein (MCP) and CR1, has an important role [7], even in the nonvascularized cell transplantation. From the result that PICs survived in the allogeneic pig serum, it may be suggested that complement regulatory proteins existing on the surface of PICs could protect an attack of complement most effectively for allogenic complement, but ineffectively for xenogeneic complement. In addition, inhibitory action by the complement regulatory proteins may be rarely performed to the complement of the dog. This specificity of complement reguratory proteins to complement may account for the difference in cytotoxicity between species.

The protection of xenotransplanted PICs from complement action of a recipient is inevitable for the successful graft survival. From the result of this study, it may be more difficult for the successful pig-to-dog islet transplantation than the pig-to-human one. Methods to protect the xenotransplanted cell from complement-mediated reaction should be needed for the development of islet xenotransplantation.

\section{REFERENCES}

1. Edamura, K., Ohgawara, H., Nasu, K., Iwami, Y., Sato, A., Ishikawa, S., Matsuki, N., Ono, K., Ogawa, H. and Sasaki, N. 2001. Cell Transplant. 10: 493-498.

2. Galili, U. 1993. Immunol. Today 14: 480-482.

3. Kato, H., Inoue, T., Ishii, N., Murakami, Y., Matsumura, M., Seya, T. and Wang, P. 2002. Biotechnol. Bioprocess Eng. 7: $67-75$.

4. Kin, T., Nakajima, Y., Aomatsu, Y., Kanehiro, H., Hisanaga, M., Ko, S., Ohyama, T. and Nakano, H. 2000. Surg. Today 30: 821-826.

5. Mayer, M. M. 1961. Complement and complement fixation. p. 133. In: Experimental Immunochemistry (Kabat, E. A. and Mayer M. M. eds), Thomas, Springfield

6. McKenzie, I. F., Koulmanda, M., Mandel, T. E. and Sandrin, M. S. 1998. J. Immunol. 161: 5116-5119.

7. Miyagawa, S., Hirose, H., Shirakura, R., Naka, Y., Nakata, S., Kawashima, Y., Seya, T., Matsumoto, M., Uenaka, A. and Kitamura, H. 1988. Transplantation 46: 825-830.

8. Ohgawara, H., Kobayashi, A., Chong, S. J., Akaike, T. and Hashimoto, Y. 1994. Cell Transplant. 3: 325-331.

9. Oriol, R., Ye, Y., Koren, E. and Cooper, D. K. 1993. Transplantation 56: 1433-1442.

10. Parker, W., Saadi, S., Lin, S. S., Holzknecht, Z. E., Bustos, M. and Platt, J. L. 1996. Immunol. Today 17: 373-378.

11. Sato, S., Ohgawara, H., Katagiri, N., Nakagawa, Y., Aikawa, E., Omori, Y. and Ota, K. 1997. Pancreas 14: 400-406.

12. Schaapherder, A. F., Wolvekamp, M. C., te Bulte, M. T., Bouwman, E., Gooszen, H. G. and Daha, M. R. 1996. Transplantation 62: 29-33. 